\title{
ALTERAÇÕES ALIMENTARES E PONDERAIS DOS USUÁRIOS COM DEPRESSÃO DE UM CAPS DO NOROESTE GAÚCHO
}

\author{
FOOD AND WEIGHT CHANGES OF PATIENTS WITH DEPRESSION \\ AT CAPS IN THE NORTHWEST GAUCHO
}

\begin{abstract}
Nilce Coelho Peixoto, ${ }^{1}$ Alana Cristina Favaretto ${ }^{2}$
${ }^{1}$ Docente do Departamento de Ciências da Saúde (Campus Palmeira das Missões), Doutora em Bioquímica Toxicológica (Curso de Pós-graduação em Bioquímica Toxicológica, Centro de Ciências Naturais e Exatas, Campus Sede)

${ }^{2}$ Acadêmica do Curso de Residência Multiprofissional Integrada em Sistema Público de Saúde (Centro de Ciências da Saúde, Campus Sede), Nutricionista (Curso de Graduação em Nutrição, Campus Palmeira das Missões)

Universidade Federal de Santa Maria/Brasil
\end{abstract}

Autor correspondente: Nilce Coelho Peixoto

e-mail: ncpeixoto@yahoo.com.br

\section{EDITORES}

Thiago Gomes Heck

(Unijuí-Brasil)

Adriane Cristina Bernat Kolankiewicz

(Unijuí-Brasil)

\section{EDITORES DE ÁREA}

Educação \& Saúde

Eva Teresinha de Oliveira Boff

(Unijuí-Brasil)

Fisioterapia \& Saúde

Eliane Roseli Winkelmann

(Unijuí-Brasil)

Ciências Farmacêuticas \& Saúde

Marilei Uecker Pletsch

(Unijuí-Brasil)

Nutrição \& Saúde

Lígia Beatriz Bento Franz

(Unijuí-Brasil)

Nadia Oliveira

(Unipampa-Brasil)

Ingrid Perry

(UNESC-Brasil)

Enfermagem e suas contribuições para a prática

Adriane Cristina Kolankiewicz

(Unijuí-Brasil)

Crhis de Brum

(UFFS-Brasil)

Neila de Souza

(UFSM-Brasil)

Exercício Físico \& Saúde

Thiago Gomes Heck

(Unijuí-Brasil)

Anderson Zampier Ulbrich

(UFPR)

Editora Unijuí

Universidade Regional do Noroeste do

Estado do Rio Grande do Sul (Unijuí)

\section{RESUMO}

O objetivo deste trabalho foi verificar os hábitos alimentares dos sujeitos com diagnóstico de depressão atendidos em um centro de atenção psicossocial (CAPS) do Noroeste do RS e identificar se existem alterações de apetite e/ou peso corporal após o surgimento da patologia. $\mathrm{O}$ estudo foi desenvolvido por meio da aplicação de uma entrevista individual contendo 17 questões objetivas e descritivas com os frequentadores com síndromes depressivas, usuários de um CAPS de Palmeira das Missões/RS. A imensa maioria dos sujeitos participantes deste estudo é do sexo feminino. Segundo os seus próprios relatos, após a manifestação da doença, muitos abandonaram o hábito de ingerir alimentos saudáveis à nutrição humana, aderiram ao consumo de doces, realizam poucas refeições diariamente e relataram alteração no peso corporal. Grande parte assumiu que frutas e verduras não fazem parte da sua dieta sistematicamente. Os fármacos mais utilizados são os ansiolíticos/hipnóticos e os antidepressivos ISCS. O ganho de peso pode estar relacionado ao consumo exagerado de alimentos hipercalóricos e à orexia. Enquanto que a perda ponderal, relacionada à sonolência, consequência do uso de hipnótico/sedativo, de menor tempo em atividade e de pequeno número de refeições feitas. Assim, sugere-se que as várias mudanças ponderais relatadas por mais de $80 \%$ dos sujeitos estudados podem estar relacionadas aos hábitos alimentares adotados e aos fármacos usados após o acometimento pela enfermidade. Conclui-se que as doenças depressivas alteram os hábitos alimentares e o peso corporal dos sujeitos. Estudos para determinar o consumo alimentar e a avaliação nutricional destes indivíduos, entretanto, são necessários para corroborar os resultados aqui encontrados.

Palavras-chave: Depressão, Fármacos, Alimentação.

Submetido em: 20/3/2016

Aceito em: 6/10/2016 


\section{Revista}

\section{Contexto}

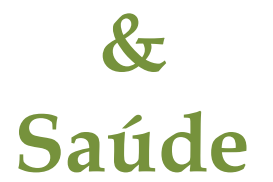

Volume 16

Número 31

2016

ISSN 2176-7114

A Revista Contexto \& Saúde é um periódico do Departamento de Ciências da Vida da Universidade Regional do Noroeste do Estado do Rio Grande do Sul (Unijuí). É um periódico semestral que tem por objetivo a divulgação da produção técnico-científica de temas relacionados à área de Ciências da Saúde.

O escopo da revista abrange a divulgação de resultados de pesquisa que contemplem avanços no processo saúde-doençacuidado e no conhecimento e aplicabilidade de novos processos químicos e biológicos em saúde.

Neste periódico, entende-se que a publicação de estudos com os aspectos epidemiológicos, assistenciais e educacionais em saúde, experimentais e aplicados é uma forma a subsidiar e qualificar a atenção à saúde de modo interdisciplinar.

\begin{abstract}
The goal of this study was to investigate the dietary habits of the subjects diagnosed with depression under treatment at a psychosocial support center in the northwestern of RS and identify if there are changes in appetite and/or body weight after the onset of the disease. The study was developed by applying an individual interview containing 17 objective and descriptive issues with the regulars with depressive syndromes of Palmeira das Missões/RS. The vast majority of the subjects participating in this study are female. According to selfreports, after the outbreak of the disease, many have abandoned the habit of eating healthy foods for human nutrition, joined the consumption of sweets, consume few meals daily and reported change in body weight. Most assumed that fruits and vegetables are not part of the diet systematically. The most commonly used drugs are anxiolytics/hypnotics and antidepressants SSRI. Weight gain may be related to overconsumption of high-calorie foods and orexia. While weight loss, drowsiness, is consequence of the use of hypnotic/sedative, inactivity, and the small number of meals. Thus, it is suggested that the various weight changes reported by more than $80 \%$ of the subjects studied adopted may be related to eating habits and drugs used after the disease onset. It follows that depressive illness changes dietary habits and body weight of the subjects. However, studies to determine the food consumption and nutritional evaluation of these individuals are needed to confirm the present results.
\end{abstract}

Keywords: Depression. Drugs. Dietary habits. 


\section{INTRODUÇÃO}

A depressão é um transtorno cerebral do humor provocado pela deficiência de neurotransmissores, moléculas que atuam no sistema nervoso central, considerado responsável pelo estado de humor. Suas consequências são prejuízos sociais e ocupacionais, que podem culminar com incapacidade e morte, uma vez que o humor deprimido acarreta em decréscimo do interesse ou prazer do paciente em executar atividades que, anteriormente à patologia, eram agradáveis. Adicionalmente, exibe sinais e sintomas como insônia, tristeza, dano na psicomotricidade, na concentração e na libido, anorexia ou hiperorexia, fadiga, prostração, culpa e pessimismo exacerbados, indecisão, pensamentos recorrentes de morte e planos de suicídio (RANG et al., 2007; GOLAN et al., 2009; SAFANELLI; RODRIGUES; CYRINO, 2012). A depressão acomete tanto as mulheres quanto os homens (APÓSTOLO et al., 2011; SILVA et al., 2012; LIMA; VIEGAS, 2011). O tratamento mais eficaz e que pode ser considerado o mais importante para a depressão é a utilização de fármacos. Atualmente, existem no mercado várias classes de drogas antidepressivas, mas uma grande porcentagem dos usuários não apresenta resposta medicamentosa adequada (BAROZA; DA SILVA, 2012; ROCHA et al., 2013).

$\mathrm{O}$ estado nutricional dos indivíduos com depressão pode sofrer alterações, uma vez que a doença se encontra fortemente associada à ansiedade e ao estresse, e estes distúrbios, relacionados com modificações do apetite, condicionam as preferências alimentares. As alterações do apetite associadas à depressão podem manifestar-se tanto como aumento ou diminuição do consumo alimentar. Os distúrbios do humor e o estresse nos indivíduos deprimidos podem influenciar na quantidade e na qualidade dos alimentos ingeridos (FRANÇA et al., 2012; BITTENCOURT et al., 2012).

Os hábitos alimentares, conjuntamente com outras variáveis modificáveis ou não, são importantes quando se pensa em um estilo de vida saudável, que influencia a saúde, a morbidade e a mortalidade (MALTA et al., 2011; AZEVEDO et al., 2014; ORLANDO et al., 2013). Alguns constituintes dos alimentos sofrem transformações metabólicas e geram neurotransmissores (LANDEIRO; QUARANTINI, 2011). O principal responsável pela sensação de bem-estar e bom humor é o neurotransmissor serotonina. De acordo com alguns autores, todavia, há variáveis que podem alterar a produção e a liberação desta substância. Quando os sinais, que são enviados ao sistema nervoso central, estão em desequilíbrio, quer por deficiência nutricional, quer por distúrbios fisiológicos, psicológicos ou psiquiátricos (como as enfermidades depressivas), provocam uma deficiência na produção de serotonina. Este fator pode vir a desencadear diversas alterações em nosso organismo, como depressão, ansiedade, aumento de peso corporal e fadiga (LANDEIRO; QUARANTINI, 2011, RAPOSO, 2010).

A alteração do peso também pode ter associação com os psicofármacos utilizados pelos pacientes. Dependendo dos neurotransmissores envolvidos em sua farmacodinâmica, eles podem influenciar na diminuição ou aumento do mesmo. Raposo (2010) apresenta uma relação de fármacos e sua ação sobre o peso corporal dos usuários. Os medicamentos que aumentam o peso são os antidepressivos (do tipo tricíclicos, mirtazapina e paroxetina), os estabilizadores de humor (carbamazepina, lítio e valproato) e os antipsicóticos (clozapina, clorpromazina, olanzapina, quetiapina e risperidona). Entre os que mantêm o peso corporal estão os antidepressivos [duloxetina, venlafaxina e outros inibidores seletivos da recaptação/captação da serotonina (ISRS/ISCS)], o estabilizador de humor lamotrigina e os antipsicóticos (aripiprazol, haloperidol e ziprazidona), enquanto as opções entre os que diminuem o peso são em menor número e correspondem aos antidepressivos (bupropiona e fluoxetina) e o estabilizador do humor topiramato (RAPOSO, 2010).

Considerando que o tratamento com maior índice de êxito para esta enfermidade é o emprego de medicamentos que, muitas vezes, têm efeitos adversos que alteram o apetite, é importante avaliar o comportamento alimentar e as alterações de peso corporal nos usuários destes fármacos. Assim, é relevante enfatizar que o excesso de peso, isoladamente ou associado a outros fatores, pode acarretar futuramente o desenvolvimento de doenças crônicas 
não transmissíveis, como hipertensão arterial, diabetes, entre outras (AZEVEDO et al., 2014; ORLANDO et al., 2013). Também o peso abaixo dos limites de eutrofia pode levar ao desenvolvimento de carências e outras deficiências nutricionais, como marasmo e Kwashiorkor (MAHAN; ESCOTT-STUMP, 2005).

O objetivo do presente trabalho foi analisar os hábitos alimentares de indivíduos com diagnóstico de depressão atendidos em um centro de atenção psicossocial (CAPS), localizado no Noroeste do Estado do Rio Grande do Sul, no município de Palmeira das Missões, e identificar se existem alterações de apetite e/ou peso corporal após o aparecimento e tratamento da patologia.

\section{MATERIAIS E MÉTODOS}

Trata-se de um estudo transversal que se utilizou de uma amostra de conveniência. Participaram da investigação os sujeitos com síndrome depressiva usuários dos serviços de saúde de um CAPS do município de Palmeira das Missões, Noroeste do Estado do Rio Grande do Sul, de novembro de 2014 a fevereiro de 2015.

Os indivíduos que fizeram parte do estudo foram arrolados pelo CAPS e identificados, por intermédio de prontuários médicos, como portadores de doença depressiva. Posteriormente, foram contatados pessoalmente, uma vez que foram buscados e localizados nos dias em que tinham consulta agendada neste centro de atendimento, ou por meio de visita domiciliar, como forma de otimizar o trabalho, e convidados a contribuir com o estudo. Estes foram esclarecidos de que sua participação consistiria em responder a um questionário que seria aplicado no formato de entrevista feita pelo pesquisador. O questionário, elaborado pelos autores, englobava 17 perguntas objetivas ou descritivas relacionadas com dados sociodemográficos, hábitos alimentares, alteração de peso corporal e medicamentos empregados. Todos os sujeitos em tratamento neste CAPS, que foram listados e nomeados ao conhecimento do pesquisador, concordaram com sua participação voluntária no presente trabalho que fazia parte de um projeto de pesquisa vinculado ao Departamento de Ciências da Saúde, do campus Palmeira das Missões/RS, da Universidade Federal de Santa Maria.

Os critérios de inclusão utilizados para classificar quais indivíduos participariam da pesquisa foram: ter diagnóstico de síndrome depressiva, estar em tratamento no CAPS do município de Palmeira das Missões/RS, residir na área urbana e concordar voluntariamente em participar.

Foram adotados como critérios de exclusão: todo e qualquer sujeito que não tivesse com diagnóstico depressivo, mesmo que em tratamento por algum outro motivo no CAPS, os residentes na área rural, pela dificuldade de se chegar até os mesmos, e os demais que se enquadravam nas normas de inclusão, mas se recusaram a participar.

Algumas entrevistas foram realizadas nas próprias dependências do CAPS nos intervalos das consultas, e as demais, em virtude do tempo escasso e para agilizar o processo de obtenção dos dados, foram feitas nas residências dos usuários do CAPS após autorização expressa dos mesmos. O questionário "Hábitos alimentares de pacientes deprimidos", elaborado pelos autores do projeto de pesquisa, foi aplicado com o intuito de esclarecer as questões propostas e alcançar o objetivo do estudo. O mesmo era composto de 17 perguntas, 13 questões objetivas e 4 descritivas. O questionário foi aplicado em um espaço de tempo aproximado de 10 a 15 minutos. Os dados derivados das entrevistas foram digitados em uma planilha de dados do Excel 2010 e os resultados estão apresentados como frequências percentuais.

Todos os participantes foram esclarecidos sobre o sigilo das informações prestadas, que sua participação era totalmente voluntária, que haveria a possibilidade de esclarecerem dúvidas em relação ao projeto de pesquisa e que teriam liberdade de desistirem do estudo a qualquer momento e sem qualquer ônus. Todos os participantes assinaram um termo de consentimento livre e esclarecido. 
Este projeto foi aprovado pelo Comitê de Ética em Pesquisa da Universidade Federal de Santa Maria, sob o número 748.218, no dia 12 de agosto de 2014.

\section{RESULTADOS}

Foram avaliadas 16 pessoas com distúrbios depressivos diagnosticados, considerando que $100 \%$ dos entrevistados estavam em tratamento psicológico e/ou medicamentoso no CAPS no período em que o estudo foi realizado, que incluiu o mês de novembro de 2014 até o mês de fevereiro do ano seguinte, totalizando quatro meses. A imensa maioria dos sujeitos estudados era do sexo feminino (93,75\%). Mais do que a metade dos entrevistados, $62,5 \%$, relatou não ter emprego fixo. Os dados etários e de escolaridade dos indivíduos estão demonstrados na Tabela 1. Cerca de metade das pessoas entrevistadas, 56,25\%, tinha idade entre 41 e 60 anos. O grau de instrução que prevaleceu entre o grupo foi o de Ensino Médio (46,67\%), e dos 16 questionados, um deles não respondeu à esta pergunta.

Dois participantes $(12,5 \%)$ relataram fazer somente terapia psicológica, sem utilizar terapia medicamentosa. O restante $(87,5 \%)$ utilizava fármacos, e a maioria deles respondeu que estes medicamentos são fornecidos pelo Sistema Único de Saúde (56\%) e, dentre os demais, a aquisição é de forma onerosa.

Tabela 1 - Dados demográficos dos sujeitos com depressão em tratamento no CAPS de Palmeira das Missões/RS durante o período de novembro de 2014 até fevereiro de 2015

\begin{tabular}{lcc}
\hline Idade (anos) & $\mathbf{N}^{\circ}$ & $\%$ \\
\hline $20-30$ & 3 & 18,75 \\
\hline $31-40$ & 2 & 12,50 \\
\hline $41-50$ & 5 & 31,25 \\
\hline $51-60$ & 4 & 25,00 \\
\hline Mais de 60 & 2 & 12,50 \\
\hline Escolaridade & $\mathbf{N}^{\circ}$ & $\%$ \\
\hline Analfabeto & 1 & 6,67 \\
\hline Até o $4^{\circ}$ ano & 4 & 26,67 \\
\hline Do 5 ao $9^{\circ}$ ano & 2 & 13,33 \\
\hline Ensino Médio & 7 & 46,67 \\
\hline Ensino Superior & 1 & 6,67 \\
\hline
\end{tabular}


No Gráfico 1 estão representadas as classes de drogas utilizadas pelos participantes, posto que os medicamentos classificados como sendo "outros" são aqueles cuja indicação clínica não está relacionada com ansiedade, depressão e psicoses. Uma das entrevistadas relatou utilizar medicamentos, mas não soube citá-los. A totalização, soma dos sujeitos que faz uso de medicamentos e que os arrolou, que são 13, é maior do que 100\%, porque grande parte utiliza mais do que um fármaco simultaneamente. Dentre estes 13 indivíduos, 7 utilizam rotineiramente mais do que um tipo de substância do tipo antidepressivo, neuroléptico e ansiolítico/hipnótico. As drogas antidepressivas ISCS e as hipnóticas/tranquilizantes estão entre as mais empregadas no tratamento medicamentoso das doenças pelas quais estão acometidos estes indivíduos, totalizando oito deles. Assim, isto corresponde a $61,5 \%$ dos que souberam enumerar as suas medicações. Ainda, é importante enfatizar que muitos participantes (6) usam regularmente outros tipos de medicamentos cuja finalidade terapêutica não é tratar a enfermidade em questão. Entre estes estão o metoprolol, o enalapril, o captopril, a hidroclorotiazida, o omeprazol, a ciclobenzaprina e o AAS.

Gráfico 1- Medicamentos utilizados pelos sujeitos com depressão em tratamento no CAPS de Palmeira das Missões/RS durante o período de novembro de 2014 até fevereiro de 2015

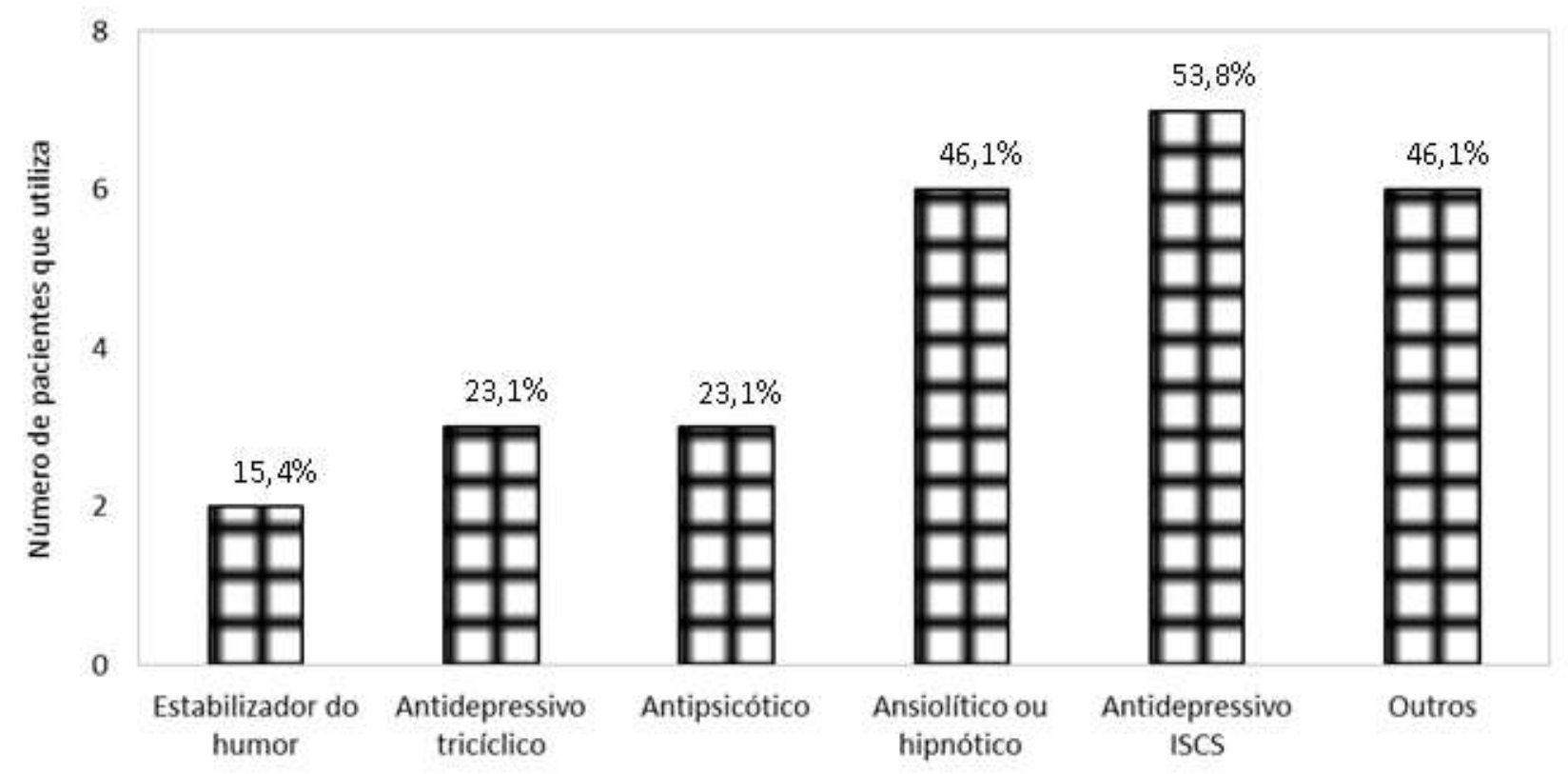

O Gráfico 2 ilustra a manutenção ou a alteração do peso corporal dos usuários estudados após o início da utilização dos fármacos prescritos para o tratamento da enfermidade depressiva. Dos entrevistados, $81 \%$ relataram alteração de peso por consequência de modificação do apetite. Destes, 44\% relataram aumento ponderal, e o restante, diminuição ponderal. A média e o desvio-padrão de ganho de peso corporal foram de $13,57 \pm 10,69 \mathrm{~kg}$ e a de perda foi de $9,66 \pm 6,25 \mathrm{~kg}$. 
Gráfico 2 - Manutenção ou alteração do peso corporal dos sujeitos com depressão em tratamento no CAPS de Palmeira das Missões/RS durante o período de novembro de 2014 até fevereiro de 2015

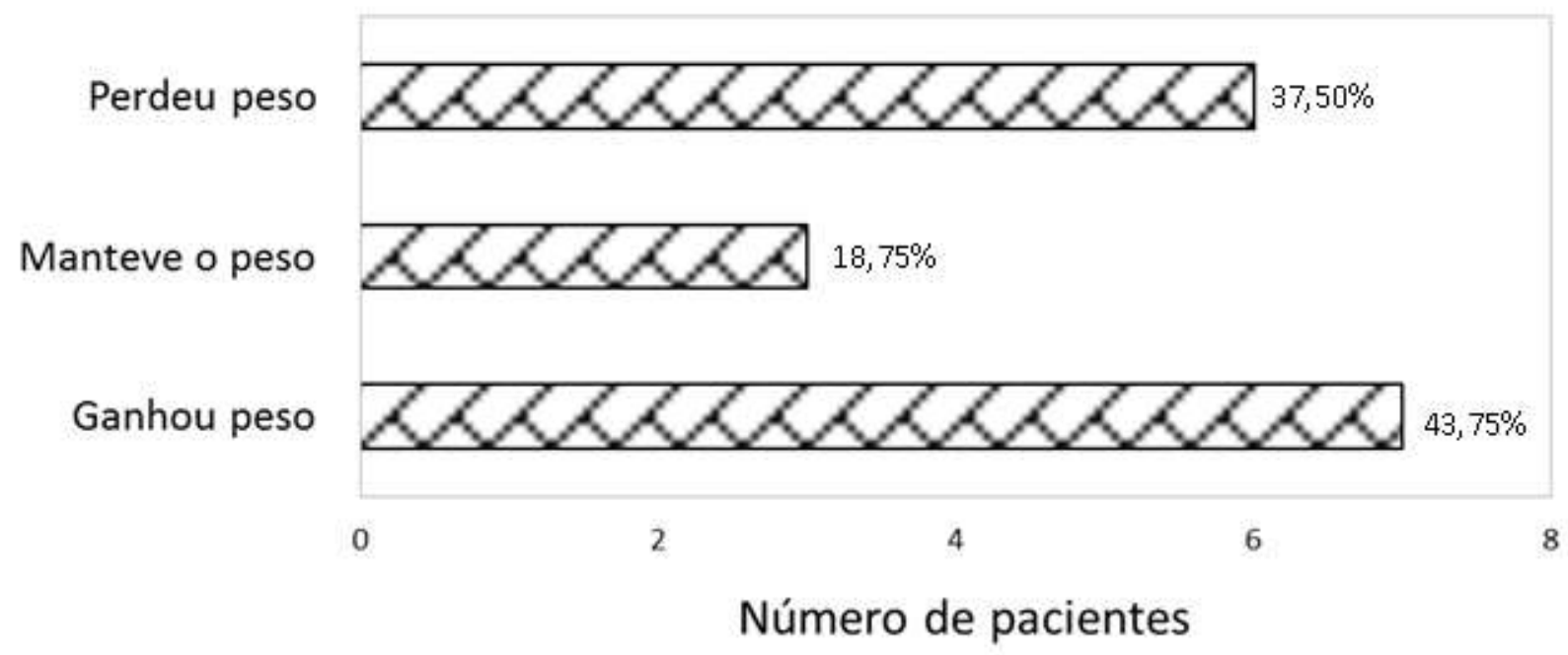

A Tabela 2 apresenta os dados relacionados aos hábitos alimentares das pessoas que responderam à entrevista. Dentre os indivíduos avaliados, $25 \%$ se alimentam uma vez ou nenhuma vez ao dia. Outros $25 \%$ fazem somente duas refeições diárias. O participante que referiu não fazer nenhuma refeição por dia relatou desta forma porque nos dias que teve episódios da doença o mesmo informou que não comia nada. Dos demais participantes, que totalizam $50 \%$, somente $37,5 \%$ fazem quatro ou mais refeições diárias. Cinco indivíduos alegaram consumir uma quantidade menor de alimentos por ter perdido a vontade de se alimentar, e um deles relatou que reduziu bruscamente o consumo alimentar porque atribui à alimentação a culpa por estar com a patologia depressiva.

A maioria dos entrevistados não consome verduras (hortaliças e legumes) e frutas de forma diária, totalizando cerca de $60 \%$ da amostra. Seis dos entrevistados relataram que, após a manifestação da doença, passaram a ingerir algum tipo de alimento que não costumavam ingerir. Destes, todos referiram-se a algum tipo de doce, e o chocolate foi o mais citado (50\% dos que passaram a consumir algum alimento que antes não consumia), seguido pelos doces em geral (33\%) e o sorvete, que contribuiu com uma porcentagem de $17 \%$. 
Tabela 2 - Hábitos alimentares dos sujeitos com depressão em tratamento no CAPS de Palmeira das Missões/RS durante o período de novembro de 2014 até fevereiro de 2015

\begin{tabular}{|c|c|c|}
\hline Número de refeições diárias & $\mathbf{N}^{\circ}$ & $\%$ \\
\hline Nenhuma & 1 & 6,25 \\
\hline Uma & 3 & 18,75 \\
\hline Duas & 4 & 25,00 \\
\hline Três & 2 & 12,50 \\
\hline Quatro & 3 & 18,75 \\
\hline Cinco & 1 & 6,25 \\
\hline Seis ou mais & 2 & 12,50 \\
\hline Consumo diário de frutas & $\mathbf{N}^{\circ}$ & $\%$ \\
\hline Sim & 6 & 37,50 \\
\hline Não & 10 & 62,50 \\
\hline Consumo diário de verduras & $\mathbf{N}^{\circ}$ & $\%$ \\
\hline Sim & 6 & 37,50 \\
\hline Não & 10 & 62,50 \\
\hline Alimentos que passaram a ser ingeridos & $\mathbf{N}^{\circ}$ & $\%$ \\
\hline Sorvete & 1 & 17,00 \\
\hline Doces em geral & 2 & 33,00 \\
\hline Chocolate & 3 & 50,00 \\
\hline Alimentos que deixaram de ser consumidos & $\mathbf{N}^{\circ}$ & $\%$ \\
\hline Carne & 4 & 44,00 \\
\hline Frutas & 3 & 33,00 \\
\hline Pão & 1 & 11,00 \\
\hline Feijão & 1 & 11,00 \\
\hline
\end{tabular}


Em razão da patologia, nove sujeitos relataram perda da vontade de se alimentar com algum alimento específico, e a carne foi o mais citado (44\% dos que passaram a preterir algum alimento que antes ingeria), seguida pelas frutas (33\%), pão e feijão (que contribuem com 11\% cada um deles).

Em relação ao consumo compulsivo de alimentos, foi questionado aos entrevistados se eles costumavam levantar à noite para se alimentar e a maioria deles relatou que não levantam neste período do dia para comer (75\%), e os demais (25\%) disseram que despertam durante o período noturno para se alimentar. Levando em conta as duas pessoas que responderam fazer em média mais de seis refeições diárias, somente uma delas levanta à noite para se alimentar. Um dos participantes, que respondeu que habitualmente faz uma refeição diária, relatou ingerir alimentos à noite.

\section{DISCUSSÃO}

A depressão é considerada um transtorno com causas múltiplas, dentre elas as psicológicas (SANTOS; SPEROTTO; PINHEIRO, 2011), as bioquímicas (ALMEIDA et al., 2013, PIRES; FREITAS; RODRIGUES, 2013) e as culturais (MARTIN et al., 2012). As frequentes alterações hormonais vitimizam mais o sexo feminino. Esta maior susceptibilidade foi observada neste trabalho, quando quase a totalidade da amostra estudada foi constituída por mulheres. Isto está de acordo com outras investigações, nas quais foi constatado que a depressão atinge mais as mulheres do que os homens (APÓSTOLO et al., 2011; SILVA et al., 2012; LIMA; VIEGAS, 2011). É importante salientar, entretanto, que os indivíduos que foram objeto dos estudos de Apóstolo et al. (2011) e de Lima e Viegas (2011) eram aqueles que buscavam auxílio em serviços de saúde. Assim, não é possível provar que o gênero masculino é menos acometido pela enfermidade em questão comparativamente às mulheres, mas estas últimas são as que mais procuram atendimento para o tratamento. A alta taxa de escolarização dos participantes, maioria com Ensino Médio completo ou mais, e com faixa etária mais frequente entre 41 e 60 anos, são fatores que podem determinar uma maior susceptibilidade à depressão. Um maior nível de instrução está subentendido, e, provavelmente, trabalham fora de casa e estão sujeitos, adicionalmente, ao estresse do trabalho, além daquele da vida doméstica, e também pode ser um determinante para a procura de auxílio e tratar a doença, uma vez que os presentes indivíduos são usuários de um serviço de atendimento e apoio em saúde. A idade destas pessoas acometidas pela patologia, neste estudo investigada, coincide com uma faixa em que há uma grande frequência de enfermidades psiquiátricas relatada por vários outros trabalhos publicados (APÓSTOLO et al, 2011; LIMA; VIEGAS, 2011; MARTIN et al, 2012).

Considerando estas prerrogativas da frequência alimentar, menos do que $40 \%$ dos entrevistados faz quatro ou mais refeições ao dia, e das opções alimentares somente uma porcentagem similar delas consome frutas, hortaliças, verduras e legumes cotidianamente. Sabe-se que o recomendado é realizar três refeições principais e três lanches saudáveis nas refeições intermediárias, evitar refrigerantes e sucos industrializados, bolos, biscoitos doces e recheados, sobremesas e outras guloseimas, para se ter uma alimentação equilibrada e um peso saudável (BRASIL, 2014). A maioria dos entrevistados não segue estas orientações já que realizam somente três refeições ou menos durante todo um dia. Estes, além disto, ou fazem estas refeições em uma quantidade mínima ou de forma exagerada. Os que realizam as seis refeições diárias relatam ter aumentado o peso corporal, sugerindo que não as fazem de forma saudável, consumindo quantidades significativas de alimentos hipercalóricos, excluindo frutas e verduras e incluindo doces e chocolates no cardápio diário. 
O aumento ponderal, relatado por quase a metade dos sujeitos estudados, pode estar relacionado ao consumo de alimentos hipercalóricos. Sete pessoas afirmaram ter aumentado o peso em um espaço de tempo curto, cinco dos indivíduos entrevistados relataram comer doces todos os dias e, destes cinco, quatro não consome nem frutas tampouco verduras diariamente. $\mathrm{O}$ ato de levantar à noite para se alimentar nem sempre coincide com $\mathrm{O}$ aumento de peso corporal, pois das quatro pessoas que o fazem duas não relataram ganho de peso, conforme os depoimentos colhidos destes sujeitos.

O consumo alimentar exagerado tem sido relacionado à obesidade, não somente quanto ao volume da ingestão alimentar, mas também quanto à composição e qualidade da dieta que é consumida. Em indivíduos depressivos o nível de serotonina geralmente está diminuído (PIRES; FREITAS; RODRIGUES, 2013), o que pode explicar o consumo exacerbado de doces entre estes sujeitos. A serotonina desempenha um importante papel no sistema nervoso, com diversas funções, como a liberação de alguns hormônios, regulação do sono, temperatura corporal, apetite, humor, atividade motora e funções cognitivas (LANDEIRO; QUARANTINI, 2011). Alterações nos níveis deste neurotransmissor têm sido relacionadas ao aumento do desejo de ingerir doces e carboidratos. Com quantidades normais de serotonina, a pessoa atinge mais facilmente a saciedade e consegue maior controle sobre a ingestão de açúcares. Os níveis mais adequados deste neurotransmissor no cérebro dependem da disponibilidade de triptofano, aminoácido precursor da serotonina (FEIJÓ; BERTOLUCI; REIS, 2011; THOMAS, 2015). Isto está exemplificado pela ingestão de chocolate, que anteriormente à depressão não ocorria, como mencionado pela metade dos seis entrevistados que alegaram este comportamento de passar a consumir algo antes não consumido.

O uso de alguns antidepressivos também pode ser o responsável pela mudança de peso corporal. Um dos participantes estava em uso de sertralina (um ISCS), associada ao alprazolam, e relatou não ter mudado o peso corporal, embora tenha diminuído bruscamente o volume de alimentos ingeridos. Já entre os dois que estavam em uso do ISCS fluoxetina, um relatou perda e o outro ganho ponderal. O entrevistado que aumentou o peso também declarou ser tabagista até pouco tempo, justificando o efeito ao abandono da adição. Dos quatro que estavam sendo medicados com citalopram (outro ISCS) associado a outros medicamentos ou não, um relatou ter perdido, um mantido e os outros dois ter ganhado massa. Dentre os poucos fármacos antidepressivos que proporcionam uma perda de peso, estão arrolados a fluoxetina e a bupropiona (RAPOSO, 2010; DOOSTI et al., 2013; BILLES; SINNAYAH; COWLEY, 2014). Smaga et al. (2014), em suas investigações farmacológicas, demonstraram que certas drogas antidepressivas clinicamente efetivas como, por exemplo, imipramina e escitalopram, têm como alvo os receptores cerebrais do tipo canabinoides, cujo efeito estimulador do apetite está entre o rol de respostas produzidas (SAITO; WOTJAK; MOREIRA, 2010). Tal achado propõe uma explicação para o efeito sobre os aumentos ponderais referidos pelos sujeitos participantes do presente estudo.

Sugere-se que a diminuição do peso corporal, verificada em seis dos sujeitos entrevistados, geralmente não está vinculada ao emprego de fármacos clinicamente indicados para o tratamento dos distúrbios depressivos, posto que são poucos os exemplares que têm este efeito. Este achado pode ser explicado pelos seguintes fatores encontrados em nosso estudo: primeiro, a baixa frequência alimentar dos indivíduos e segundo, a utilização de medicamentos ansiolíticos, registrada em três deles. As drogas ansiolíticas são tranquilizantes que têm como efeitos colaterais a sedação e a sonolência, o que também pode corroborar para a escassa frequência alimentar, uma vez que o indivíduo passaria sob vigília um tempo menor do dia (RANG et al., 2007; GOLAN et al., 2009). Entre as pessoas que faziam poucas refeições diariamente (três ou menos) foi autorrelatada perda ponderal bastante pronunciada (até $20 \mathrm{~kg}$ em um intervalo de tempo de apenas dois meses).

Um dos entrevistados mencionou ter parado de comer, pois atribui à alimentação a "culpa" de estar doente. O consumo diário e exagerado de doces, principalmente do chocolate, já foi correlacionado à depressão com a alegação dos consumidores de que há alívio dos sintomas da enfermidade (AZIZ; AL-MUWALLAD; MANSOUR, 2011). Resultados encontrados em vários estudos apontam um consumo maior deste tipo de 
alimento em pacientes com sintomas de depressão (RAPOSO, 2010; AZIZ; AL-MUWALLAD; MANSOUR, 2011). Na presente pesquisa, a metade dos entrevistados expôs não ingerir doces diariamente, entretanto seis deles relataram que passaram a consumir guloseimas que, previamente ao diagnóstico da doença, não costumavam ingerir, e oito responderam que passaram a rejeitar alimentos importantes na dieta humana, como carne, frutas e feijão. Dos nove que deixaram de consumir algo que costumavam ingerir, cinco são aqueles que passaram a comer doces posteriormente à doença depressiva. Entre estes últimos cinco participantes, que substituíram um item saudável por outro desnecessário na sua alimentação, quatro aumentaram o peso.

Os resultados encontrados nesta investigação apontam para o fato de que as síndromes do tipo depressivas provocam alterações nos hábitos alimentares dos indivíduos em tratamento medicamentoso relacionadas à frequência e preferência e/ou rejeição. As rejeições a determinados alimentos, considerados saudáveis e importantes na alimentação humana, figuraram como um fator frequente, assim como a introdução do consumo de doces entre os que não os ingeriam habitualmente anteriormente à doença. Tais modificações supostamente são responsáveis pelas alterações de peso corporal mencionadas pela maioria dos entrevistados no presente estudo.

\section{CONCLUSÃO}

A partir desta análise pode-se concluir que o acometimento pela síndrome depressiva nos sujeitos usuários dos serviços do CAPS de Palmeira das Missões, localizado no Noroeste do Rio Grande do Sul, durante o espaço temporal estudado, prevaleceu entre indivíduos do sexo feminino. As alterações de peso corporal, tanto o ganho quanto a perda, são comuns entre os indivíduos tratados farmacologicamente para depressão, assim como os tipos de alimentos que são ou que passam a ser consumidos ou preteridos. Desta forma, o acompanhamento destas pessoas por um profissional nutricionista seria válido no sentido de instruí-las e discipliná-las quanto aos seus hábitos alimentares. Adicionalmente, são necessárias mais investigações que determinem a avaliação nutricional e o consumo alimentar dos afetados pela enfermidade depressiva.

\section{REFERÊNCIAS}

ALMEIDA, M. M. R. et al. A depressão e sua relação com o hipotireoidismo. Revista de Medicina e Saúde de Brasília, v. 2, n. 3, p. 164-168, nov. 2013.

APÓSTOLO, J. L. A. et al. Depressão, ansiedade e estresse em usuários de cuidados primários de saúde. Revista Latino-Americana de Enfermagem, v. 19, n. 2, p. 348-353, mar./abr. 2011.

AZEVEDO, E. C. C. et al. Consumo alimentar de risco e proteção para as doenças crônicas não transmissíveis e sua associação com a gordura corporal: um estudo com funcionários da área de saúde de uma universidade. Ciência \& Saúde Coletiva, v. 19, n. 5, p. 1.613$1.622,2014$.

AZIZ, N. A. H. A.; AL-MUWALlAD, O. K.; MANSOUR, E. A. K. Neurotic depression and chocolate among female medical students at College of Medicine, Taibah University Almadinah Almunawwarah, Kingdom of Saudi Arabia. Journal of Taibah University Medical Sciences, v. 6, n. 2, p. 139-147, set. 2011.

BAROZA, P. S.; DA SILVA, D. A. Medicamentos antidepressivos e antipsicóticos prescritos no Centro de Atenção Psicossocial (CAPS) do município de Porciúncula RJ. Acta Biomédica Brasiliensia, v. 3, n. 1, p. 85-97, jun. 2012.

BILLES, S. K.; SINNAYAH, P.; COWLEY, M. A. Naltrexone/bupropion for obesity: an investigational combination pharmacotherapy for weight loss. Pharmacological Research, v. 84, p. 1-11, jun. 2014. 
BITTENCOURT, S. A. et al. Anxiety and depression symptoms in women with and without binge eating disorder enrolled in weight loss programs. Trends in Psychiatry and Psychotherapy, v. 34, n. 2, p. 87-92, aug. 2012.

BRASIL. Ministério da Saúde. Secretaria de Atenção à Saúde. Departamento de Atenção Básica. Guia alimentar para a população brasileira. Segunda edição, primeira reimpressão. Brasília: Ministério da Saúde, 2014.

DOOSTI, M-H. et al. Impacts of early intervention with fluoxetine following early neonatal immune activation on depression-like behaviors and body weight in mice. Progress in Neuro-Psychopharmacology \& Biological Psychiatry, v. 43, p. 55-65, june. 2013.

FEIJÓ, F. M.; BERTOLUCI, M. C.; REIS, C. Serotonina e controle hipotalâmico da fome: uma revisão. Revista da Associação Médica Brasileira, v. 57, n. 1, p. 74-77, 2011.

FRANÇA, C. L. et al. Contributions of psychology and nutrition to the change of eating behavior. Estudos de Psicologia, v. 17, n. 2, p. 337-345, maio/ago. 2012.

GOLAN, D.E. et al. Princípios de farmacologia: a base fisiopatológica da farmacoterapia. Segunda edição. Rio de Janeiro: Guanabara Koogan, 2009.

LANDEIRO, F. M.; QUARANTINI, L. D. C. Obesidade: controle neural e hormonal do comportamento alimentar. Revista de Ciências Médicas e Biológicas, Salvador, v. 10, n. 3, p. 236-245, set./dez. 2011.

LIMA, M. S.; VIEGAS, C. A. A. Avaliação do grau de ansiedade, depressão e motivação dos fumantes que procuraram tratamento para deixar de fumar no Distrito Federal. Revista Brasileira de Cancerologia, v. 57, n. 3, p. 345-353, maio 2011.

MAHAN, L. K.; ESCOTT-STUMP, S. Alimentos, nutrição \& dietoterapia. 11. ed. São Paulo: Roca, 2005.

MALTA, D. C. et al. Fatores de risco e proteção para doenças crônicas não transmissíveis entre beneficiários da saúde suplementar: resultados do inquérito telefônico Vigitel, Brasil, 2008. Ciência e Saúde Coletiva, v. 16, n. 3, p. 2.011-2.022, 2011.

MARTIN, D. et al. Significado da busca de tratamento por mulheres com transtorno depressivo atendidas em serviço de saúde público. Interface: Comunicação, Saúde, Educação, v. 16, n. 43, p. 885-899, out./dez. 2012.

ORLANDO, R. et al. Avaliação da alimentação e sua relação com as doenças crônicas não transmissíveis de um grupo de idosos de um município da região norte do Estado do RS. Revista de Enfermagem da URI, v. 6, n. 6, p. 203-217, 2013.

PIRES, L. F.; FREITAS, R. M.; RODRIGUES, A. C. T. Efeito antidepressivo e atividade serotoninérgica da curcumina em modelos de animais de depressão. Revista de Farmacología de Chile, v. 6, n. 1, p. 37-44, 2013.

RANG, H.P. et al. Rang \& Dale: farmacologia. 6. ed. Rio de Janeiro: Elsevier, 2007.

RAPOSO, J. I. C. C. Fatores associados à variação de peso em doentes com depressão. Lisboa, Portugal. 2010. Dissertação (Mestrado) - Curso de Mestrado em Dietética e Nutrição, Universidade de Lisboa, 2010.

ROCHA, F. L. et al. Evidências sobre a combinação de antidepressivos na depressão maior. Diagnóstico e Tratamento, v. 18, n. 2, p. 87-93, 2013.

SAFANELLI, C.; RODRIGUES, J. K.; CYRINO, L. A. R. Transtorno afetivo bipolar relacionado ao uso abusivo de substâncias psicoativas: uma revisão bibliográfica. Revista Contexto \& Saúde, v. 12, n. 23, p. 15-25, jul./dez. 2012.

SAITO, V. M.; WOTJAK, C. T.; MOREIRA, F. A. Exploração farmacológica do sistema endocanabinoide: novas perspectivas para o tratamento de transtornos de ansiedade e depressão? Revista Brasileira de Psiquiatria, v. 32, Supl. I, p. 7-14, maio, 2010.

SANTOS, D. R.; SPEROTTO, D. F.; PINHEIRO, U. M. S. A medicina tradicional chinesa no tratamento do transtorno de ansiedade: um olhar sobre o stress. Revista Contexto \& Saúde, v. 10, n. 20, p. 103-112, jan./jun. 2011. 
SILVA, E. R. et al. Prevalência e fatores associados à depressão entre idosos institucionalizados: subsídio ao cuidado de enfermagem. Revista da Escola de Enfermagem da USP, v. 46, n. 6, p. 1.387-1.393, maio, 2012.

SMAGA, I. et al. Antidepressants and changes in concentration of endocannabinoids and N-acylethanolamines in rat brain structures. Neurotoxicology Research, v. 26, p. 190-206, 2014.

THOMAS, S. R. L-Tryptophan as an antidepressive agent in the management of treatment-resistant unipolar depression in borderline personality disorder: Three case reports. Advances in Integrative Medicine, v. 2, p. 68-71, 2015. 